\title{
Wideband frequency domain detection using Teager-Kaiser energy operator
}

\author{
Matthieu Gautier, Vincent Berg and Dominique Noguet \\ CEA-LETI, Minatec, Grenoble, France \\ matthieu.gautier@irisa.fr ${ }^{1}$, vincent.berg@cea.fr, dominique.noguet@cea.fr
}

\begin{abstract}
This paper addresses wireless microphone sensing in the TV white space and efficient detection of narrowband FM modulation signals. To this end, a wideband frequency domain analysis is proposed. The required Fast Fourier Transform for this operation may be shared between sensing analysis and modulation functions. A particular decision metric is then studied for the analysis of wireless microphone signals based on the Teager-Kaiser energy operator.

Simulation results show that $6 \mathrm{~dB}$ of detection gain could be achieved when using a frequency domain analysis compared to time domain methods. The Teager-Kaiser detection leads to further improvement of $1.5 \mathrm{~dB}$. This performance could be reached at no extra cost in term of complexity.
\end{abstract}

\section{INTRODUCTION}

The performance of sensing algorithms is fundamental to establish the opportunistic communication of a cognitive radio (CR) system [1]. The UHF band is a under-used candidate band where the CR system could operate [2]. In this band, the primary users are TV transmitters and wireless microphones. If the detection of TV signals has been addressed in the literature, solutions should be proposed for the detection of wireless microphones [3].

Wireless microphone signals are narrowband in comparison to their overall possible band of operation. This makes wireless microphone sensing difficult. Indeed, the European TV band is composed of 48 channels of $8 \mathrm{MHz}$ bandwidth. Each band has to be analyzed and TV and microphone signals must be detected. A statistic test should be provided to the CR system where the final decision on occupancy assessment is made. The analyzed TV channel is extremely wide compared to the frequency band occupied by the microphone signal (i.e.: around $200 \mathrm{kHz}$ ). Thus, the detection of wireless microphones may be considered a narrowband signal detection problem.

Most of the references available in the literature use a blind detection for the specific case of wireless microphones [3]. These studies are based on eigenvalue decomposition [4], spectral correlation [5] and energy detection [6]. One common property of these algorithms is that they assume the detection of wideband signals, i.e.: the analyzed bandwidth is of the same order as the signal bandwidth.

In this paper, the proposed architecture considers the narrowband property of the wireless microphone signal in the TVWS and proposes sharing a wideband Fast Fourier Transform

\footnotetext{
${ }^{1}$ Matthieu Gautier is currently an associate professor at the CAIRN team of the IRISA laboratory. The study and results presented in this paper have been carried out when he was a research engineer at CEA-LETI.
}

(FFT) operator that can be an element used by the cognitive device to simultaneously detect primary users and make the communication link. Indeed, the concept of "spectrum pooling" has been proposed as a candidate modulation scheme for CR system [7]. It proposes to use a wideband FFT to access an important bandwidth and allocate frequency bins to CR users according to white spaces opportunities.

The same FFT operation may also be used to perform the sensing step [8]. The outputs of the FFT are analyzed using specific frequency domain metrics. This leads to a wideband sensing operation and a low complexity architecture with shared FFT operation.

Furthermore, a frequency domain metric is specificaly proposed to detect wireless microphone signals. The authors proposed in [9] the Teager-Kaiser detector that allows a better estimation of the energy of the wireless microphone signal. Introduced in the time domain [9], a frequency domain counterpart of this operator is introduced and analyzed in this paper.

This paper consists of 5 parts. Section II gives the models of the wireless microphone signals and of the CR system. In Section III, the wideband frequency domain detector is introduced and its application to Teager-Kaiser energy operator is detailed. Simulation results and complexity evaluation are given in Section IV. Finally, conclusions are drawn and outlook is provided.

\section{WIRELESS MICROPHONE SENSING}

\section{A. The wireless microphone signal model}

Wireless microphones operate in the UHF band. Most of them use an analog FM modulation [3]. The signal has a spectral bandwidth $B_{x}$ of $200 \mathrm{kHz}$. But, most of the signal energy is concentrated in a bandwidth of $100 \mathrm{kHz}$. The transmit power is a few tens of $\mathrm{mW}$. The coverage area is therefore relatively low, about 500 meters for the most powerful microphones. The microphone signal, $x(t)$, may be modeled as follows:

$$
x(t)=A \cos \left(2 \pi f_{0} t+\frac{\kappa_{f}}{s_{m}} \int_{\tau} s(\tau) d \tau\right),
$$

where $f_{0}$ is the carrier frequency, $\kappa_{f}$ the frequency deviation of the FM modulation, and $s(t)$ the modulating signal having an amplitude $s_{m}$. The signal $x(t)$ has a power $\sigma_{x}^{2}$ equals to $A^{2} / 2$.

Let $y(t)$ be the microphone signal received by the opportunist 
receiver and $n(t)$ an Additive White Gaussian Noise (AWGN) with a zero mean and a variance $\sigma_{n}^{2}$ :

$$
y(t)=x(t)+n(t) .
$$

$x(t)$ and $n(t)$ being independant, the Signal to Noise Ratio $(S N R)$ received by the opportunistic user is:

$$
S N R=\frac{\sigma_{x}^{2}}{\sigma_{n}^{2}}=\frac{A^{2}}{2 \sigma_{n}^{2}} .
$$

\section{$B$. The cognitive radio system description}

The CR detector has to detect signals in the presence of noise. The problem can be described by the following hypothesis:

$$
\left\{\begin{array}{l}
H_{0}: y(t)=n(t) \\
H_{1}: y(t)=x(t)+n(t)
\end{array},\right.
$$

where $H_{0}$ is the null hypothesis for the event "free band" and $H_{1}$ is the alternative hypothesis for the event "occupied band".

By choosing one of the two assumptions $H_{0}$ and $H_{1}$, two kinds of errors can occur:

- a false alarm error: it corresponds to the case when the hypothesis $H_{1}$ is chosen while the band is free. The false alarm probability is denoted by $p_{F A}$,

- a non-detection error: it occurs when the hypothesis $H_{0}$ is chosen while the band is occupied. The non-detection probability is denoted by $p_{N D}$.

The performance of the detectors is evaluated by computing the detection probability $p_{D}\left(p_{D}=1-p_{N D}\right)$ for a given $p_{F A}$. The $p_{F A}$ imposes the value of the absolute detection threshold. The probability, $p_{D}$, is given for different powers of the received signal in order to determine the minimum $S N R$ that can be detected.

A challenge is to achieve high sensitivity at a low cost of complexity. The digital complexity is calculated in number of operations needed to provide a decision. This paper aims at proposing a detector with the best tradeoff between its detection sensitivity and its complexity.

In this study, the detection band is a TV UHF channel, its bandwidth $B_{c}$ is equal to $8 \mathrm{MHz}$ in Europe and $6 \mathrm{MHz}$ in the US.

\section{State of the art of wireless microphone detection}

Unlike the detection of digital TV signals which can use the characteristics of the Orthogonal Frequency Division Multiplexing modulation [10], wireless microphone sensing is difficult due to the few characteristics of its signal. Most of the literature references use a blind detection for the specific case of wireless microphones [3][4][5][6]. Energy and autocorrelation detections are detailed as comparison techniques for our study.

The energy detector computes a variable which is proportional to the energy of the received signal [11][12]. The test statistic $T$ of this detector is given by:

$$
T=\frac{1}{N_{s}} \sum_{k=0}^{N_{s}-1}|y[k]|^{2},
$$

where $N_{s}$ the number of samples of the analyzed signal. The autocorrelation based detection tests the stationarity of the signal by computing the samples autocorrelation function. First, the receiver estimates the autocorrelation function $C_{y}[\tau]$ of the received signal:

$$
C_{y}[\tau]=\sum_{k=0}^{N_{s}-1} y[k] y^{*}[k-\tau],
$$

where ${ }^{*}$ represents complex conjugation. Then, the following test statistic is calculated [13]:

$$
T=\frac{\sum_{\tau=0}^{N_{s}-1}\left|C_{y}[\tau]\right|^{2}}{\left|C_{y}[0]\right|^{2}} .
$$

When no signal is present, the two terms should be roughly equal, since the non-central values $(\tau \neq 0)$ should be approximately zero. When the wireless microphone signal is present, the signal is not white and the statistical test should increase.

\section{Teager-Kaiser detector}

Introduced in [9], this method is suitable to detect wireless microphones. Instead of using the conventional energy detector, we propose to use the Teager-Kaiser energy operator to measure the energy activity of a sample. This operator better reflects the energy of FM signal.

In 1990 [14], Kaiser used the results of Teager and Teager (especially the energy curve needed to produce speech) which showed the non-linear model of the speech. These changes of the characteristics of the speech signal can be modeled as a linear combination of AM-FM signals.

Based on this model, Kaiser has proposed a very simple and fast algorithm [14] to estimate the energy, called the TeagerKaiser energy operator, whenever the restriction related to the bandwidth of the signal (narrowband signal) is respected.

One of the first applications of this operator is the detection of FM modulations. The Teager-Kaiser energy operator $\Psi$ extracts directly the energy from the instantaneous signal and is expressed by:

$$
\Psi\{x[k]\}=(x[k])^{2}-x[k+1] x^{*}[k-1] .
$$

In the previous part, we have seen that the wireless microphone is a FM modulation of a speech signal, so the Teager-Kaiser detector should be adapted to this kind of signal. From (8), the semi-blind detection could be performed by computing the test statistic:

$$
\begin{aligned}
T & =E\langle\Psi\{y[k]\}\rangle, \\
& =E\langle\Psi\{x[k]\}\rangle+\sigma_{n}^{2},
\end{aligned}
$$

where $E\langle\rangle$ is the mathematical expectation operator. The main result in [9] is the sensitivity of the Teager-Kaiser detector. Taking into account the non-linear model of the FM signal energy, this operator leads to a $2 \mathrm{~dB}$ gain compared to classical energy detection without an important increase of the complexity. Indeed, the number of real multiplications needed to process $N_{s}$ samples is $N_{s}+2$ for the Teager-Kaiser detector instead of $N_{s}$ for the energy detector. 


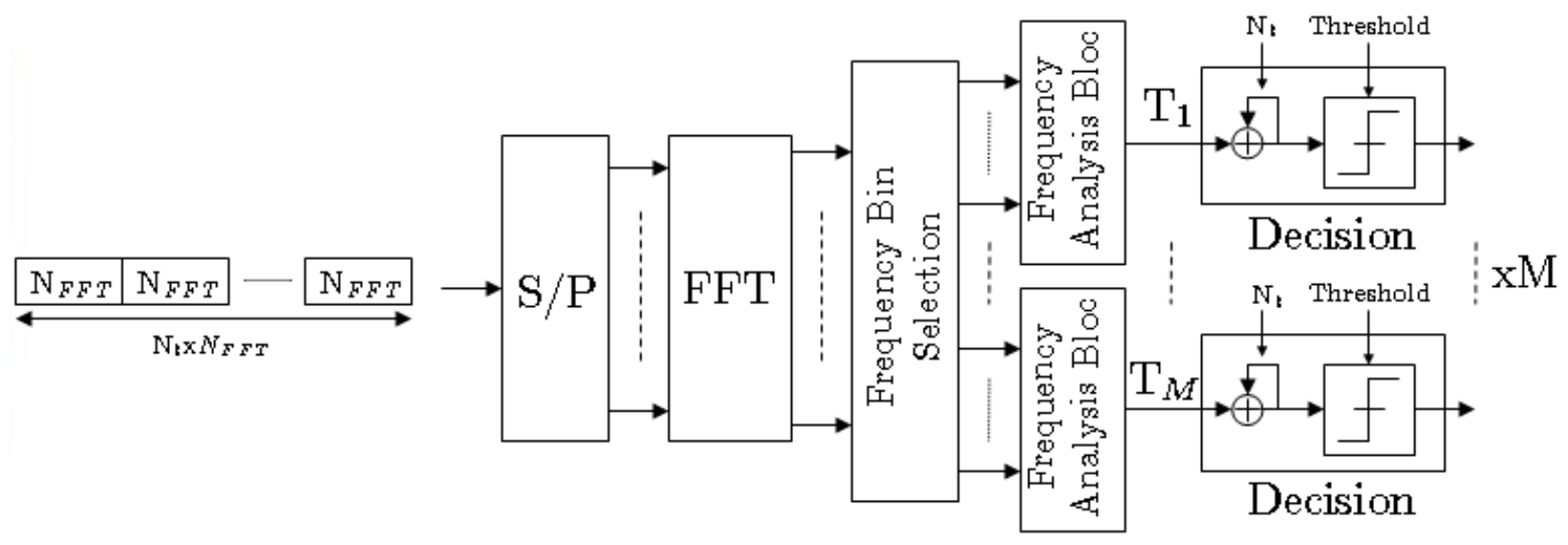

Fig. 1. Architecture of the wideband frequency domain detector.

\section{WIDEBAND FREQUENCY DOMAIN DETECTION}

The main purpose of this study is to analyse the band in the frequency domain by using a wideband FFT operator. First, the general architecture of the detector is introduced. Its application to Teager-Kaiser detection is then detailed.

\section{A. General architecture}

The proposed scheme follows the idea of spectrum pooling introduced in [7]. Proposed as a transmission technique used by an opportunist user, the idea is to match the size of the FFT used in the digital baseband of the transceiver to the possible bandwith of the application. The bandwidth of one subband should then be an integer multiple of the carrier spacing. Two key advantages can be identified for the transceiver architecture [8]. First, the input of the FFT can be fed with zeros at frequencies where incumbent users are present, while vacant subbands are used for transmission by the opportunistic radio. Second, the FFT operation required at the receiver to invert the modulation may also be used to monitor spectral activity of the other users. The proposed architecture is based on this second idea.

Fig.1 shows the general architecture of the proposed detector. It is composed of three main blocs: an FFT operation, a "Frequency Bin Selection" bloc and several "Frequency Analysis blocs".

In order to compute a large FFT, the $N_{s}$ samples of the signal are split into $N_{t}$ slots of size $N_{F F T}$ which is the size of the FFT. The outputs of the FFT operation are:

$$
Y(\nu)=\sum_{k=0}^{N_{F F T}-1} y[k] e^{-j 2 \pi \frac{\nu}{N_{F F T}} k} .
$$

The "Frequency Bin Selection" is a way of scanning the whole band, its parameters are the number of subbands $M$ and the overlapping ratio $O R$ between two adjacent bands. In that case, the number of frequency bins per subbands (denoted by
$N)$ is:

$$
N=\frac{N_{F F T}}{M(1-O R)} .
$$

Theses parameters are set according to the signal. For example, if a $40 \mathrm{MHz}$ band is analyzed and a $8 \mathrm{MHz}$ channel is sensed, $M=5$ subbands and no overlapping, $O R=0$, are needed. If wireless microphone signals are sensed in a $8 \mathrm{MHz}$ band, overlapping is needed because of the random frequency position of the signal and the bandwidth of each subband must be set to $200 \mathrm{kHz}$.

The "Frequency Analysis bloc" provides a test statistic from the frequency bins selected by the previous bloc. One bloc uses $N$ samples to compute the metric.

Several test statistics can be computed in the frequency domain. Starting with the detectors introduced in section II-C as the state of the art for wireless microphone detection, two test statistics are first introduced in the frequency domain.

The frequency domain energy detection could be directly computed from the frequency bins $Y(\nu)$. It is defined by the following metric:

$$
T_{i}=\frac{1}{N} \sum_{\nu=B_{i}}^{B_{i}+N-1}|Y(\nu)|^{2},
$$

$B_{i}=N(1-O R) i$ being the first sample of the subband $i$ $(i=0, \ldots M-1)$.

Contrary to energy detection, the autocorrelation detection must be computed in the time domain by using an IFFT of size $N$. From the frequency bins $Y(\nu)$, the autocorrelation function per subband $C_{i}(i=0, \ldots M-1)$ is computed by:

$$
C_{i}[\tau]=\sum_{\nu=B_{i}}^{B_{i}+N-1} Y[\nu] Y^{*}[\nu] e^{j 2 \pi \frac{\nu}{N} \tau} .
$$

After the frequency analysis bloc, the decision is made by averaging $N_{t}$ FFT frames. 


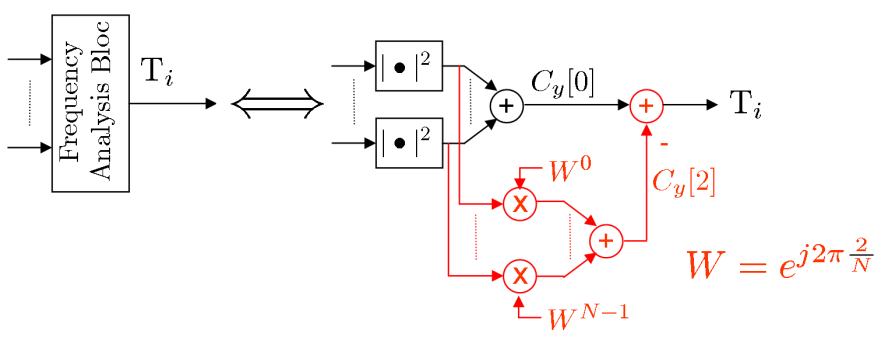

Fig. 2. Frequency analysis bloc for Teager-Kaiser detection.

\section{B. Application to Teager-Kaiser detection}

In the particular case of detection of wireless microphones as described in Section II, we propose an architecture of the Frequency Analysis bloc based on the Teager-Kaiser energy operator. Using the autocorrelation function described in (6), the Teager-Kaiser detector could be written in the time domain by:

$$
\begin{aligned}
T=E\langle\Psi\{y[k]\}\rangle & =E\left\langle y^{2}[k]\right\rangle-E\left\langle y[k+1] y^{*}[k-1]\right\rangle, \\
& =C_{y}[0]-C_{y}[2] .
\end{aligned}
$$

$C_{y}[0]$ is the energy of the signal and $C_{y}[2]$ is the tap 2 of the autocorrelation function.

So to compute the Teager-Kaiser energy operator from the FFT of the signal, the two following terms should be calculated:

$$
\begin{aligned}
C_{y}[0] & =\sum_{\nu=0}^{N-1} Y[\nu] Y^{*}[\nu], \\
\text { and } C_{y}[2] & =\sum_{\nu=0}^{N-1} Y[\nu] Y^{*}[\nu] e^{j 2 \pi \frac{\nu}{N} 2} .
\end{aligned}
$$

Fig. 2 describes the structure of the frequency analysis bloc in the case of the Teager-Kaiser detection.

\section{Architecture settings}

In the following, the wireless microphone study case is used to analyze the proposed architecture. Based on the European UHF band specifications, the bandwidth is $8 \mathrm{MHz}$. We consider that a large FFT of 4096 bins is used as a fixed parameter of the system.

The baseband microphone signal is generated following the model of (1) with these parameters: the frequency bandwidth of $s(t)$ is $20 \mathrm{kHz}$, the FM deviation $\kappa_{f}$ is 3 , the carrier frequency $f_{0}$ is equal to $100 \mathrm{kHz}$ and the noise power $\sigma_{n}^{2}$ is set to $0 \mathrm{dBm}$. Under these conditions, the transmitted microphone signal $x(t)$ has a frequency bandwidth of around $100 \mathrm{kHz}$.

The first characteristic that must be specified is the number of input samples that can be analyzed by this architecture. Indeed, according to the test statistic, a bias could appear between the time and the frequency domain computations of the metric. For example, a FFT size of $N_{F F T}$ can be used to compute the frequency domain energy of $N_{F F T}$ input samples without any bias. However, for the autocorrelation and TeagerKaiser metrics, a bias may occur depending the size of the inputs samples.

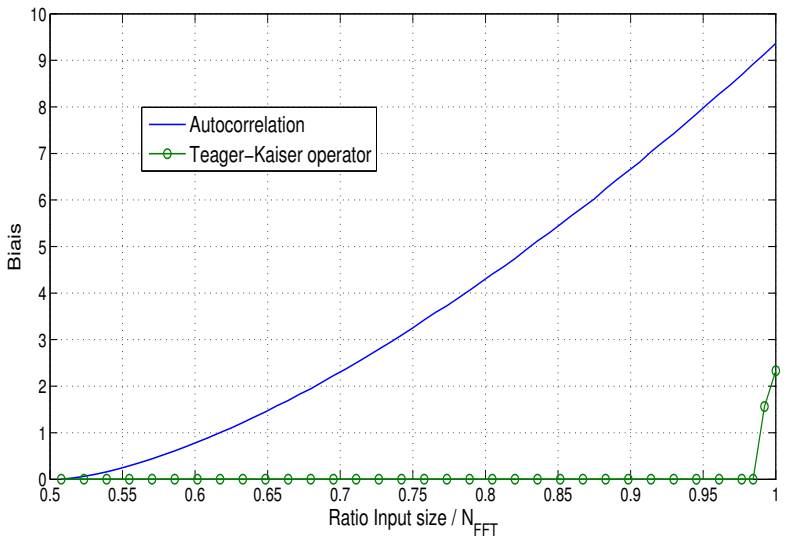

Fig. 3. Bias versus ratio between Input size and FFT size.

Fig. 3 gives the total introduced bias for both metrics as a function of the ratio between the input sample size and the FFT size. Results show that, for the autocorrelation function, a ratio of more than 0.5 induces a significant bias. The bias increases then more rapidely as the ratio is increased. For the Teager-Kaiser energy operator, the bias starts to become significant only for a ratio closed to 1 . This bias is lower than the autocorrelation for the same ratio of input size over FFT size.

This analysis outlines that the comparison of the different "Frequency Analysis bloc" is not obvious. Indeed, using a fixed FFT size, the same number of samples could not be analyzed without any bias. Thus, in the following, only the energy detection and the Teager-Kaiser detection will be computed in the frequency domain. For the Teager-Kaiser detection, 2 samples (insignificant compared to the FFT size of 4096) won't be used in order to have no bias.

In a second step, the number of subbands $M$ has to be set. Obtained by simulation, the sensitivity of the two frequency domain detectors is plotted versus the number of subbands. The sensitivity is the minimum $S N R$ that the detector could sense with $p_{D}=95 \%$ and $p_{F A}=1 \%$. The results are shown in Fig. 4 for an $O R$ of $20 \%$ and $N_{t}=1$.

Simulation results show that the sensitivity increases with the number of subchannel. For large number of subchannel, the $S N R$ decreases with a lower step and seem to reach a $S N R$ floor. This occurs when the subband bandwidth becomes smaller that the signal bandwidth. In the following, $M=16$ will be used.

\section{Performance analysis}

\section{A. Detection sensitivity}

The main feature of a detector is its sensitivity level expressed in $S N R$. Fig. 5 shows the performance of the two frequency domain algorithms. Performance is compared with the time domain algorithms. The detection probability $p_{D}$ is computed versus the $S N R$ of the wireless microphone received signal. The architecture settings are $M=16, O R=20 \%$, $N_{t}=1$ and $N_{F F T}=4096$ and the $p_{F A}$ is $1 \%$.

For a target $p_{D}$ of $95 \%$, the frequency domain detectors enable 


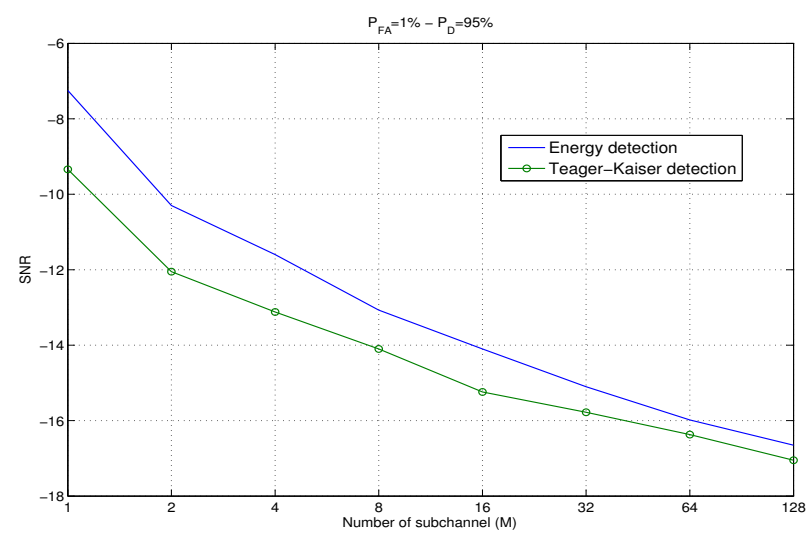

Fig. 4. Sensitivity in $S N R$ versus number of subchannel.

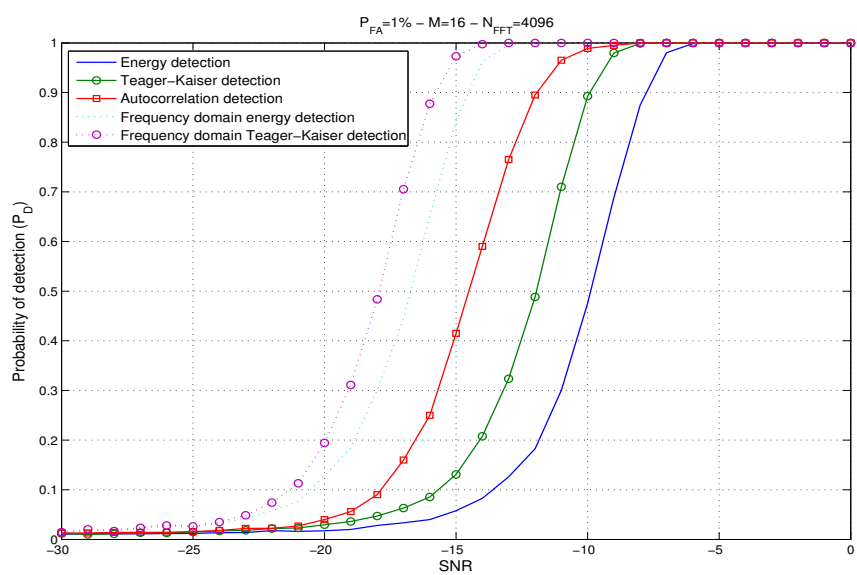

Fig. 5. Detection probability versus $S N R$.

the detection of signals with $S N R$ of $-14 \mathrm{~dB}$ and $-15.5 \mathrm{~dB}$ for the energy and the Teager-Kaiser metrics respectively. In the time domain, the sensitivity levels are $-7.5 \mathrm{~dB}$ for the energy detector, $-9.5 \mathrm{~dB}$ for the Teager-Kaiser detector and $-11.5 \mathrm{~dB}$ for the autocorrelation detector.

The simulation results show that, to narrowband the analyzed bandwidth leads to a $6 \mathrm{~dB}$ improvement of the time domain energy detection. A further $1.5 \mathrm{~dB}$ gain is achieved using the Teager-Kaiser energy operator. Both frequency domain detectors outperform the autocorrelation detector with a gain of $4 \mathrm{~dB}$ for the Teager-Kaiser detector and a gain of $2.5 \mathrm{~dB}$ for the energy detector.

\section{B. Complexity evaluation}

After the sensitivity evaluation, the complexity of the frequency domain algorithms should be evaluated and compared to their time domain counterpart. A simple way to estimate complexity is to determine the total number of real multiplications for each algorithm. Assuming split radix-2 implementation of the FFT, the number of real multiplications to process $N_{F F T}$ samples are:

- for the frequency domain energy detector:

$$
N_{F F T} * \log _{2}\left(N_{F F T}\right)+N_{F F T},
$$

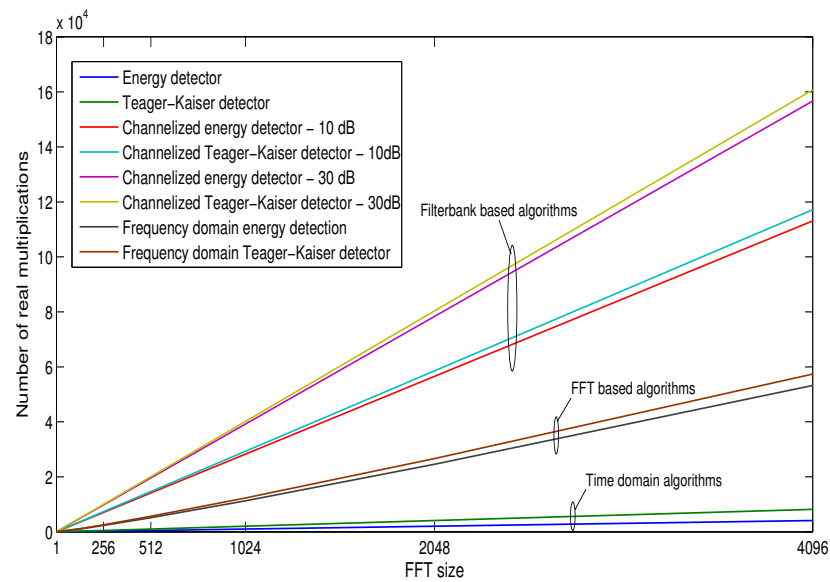

Fig. 6. Complexity in number of real multiplications versus FFT size.

- for the frequency domain Teager-Kaiser detector:

$$
\left(N_{F F T}+2\right) * \log _{2}\left(N_{F F T}+2\right)+2 *\left(N_{F F T}+2\right) .
$$

The complexity is compared with the one of a filterbank based architecture as introduced in [9]. In order to split the frequency band into subbands, an alternative to FFT is the use of a filterbank in order to channelized the wideband. To understand the requirements on the filter used by the filterbank, we derive a rejection requirement $A$ (in $\mathrm{dB}$ ) on the filter and then extrapolate the number of taps required for a finite impulse response implementation of the filter. The filter length $L_{\text {filter }}$ is estimated using an equiripple lowpass implementation. Using a low complexity dyadic implementation of the filterbank [15], the complexity of this "channelized structure" is:

$$
L_{\text {filter }} * M * \log _{2}(M) * N_{F F T} / M,
$$

to process $N_{F F T}$ samples. Then, $N_{F F T}$ and $2 * N_{F F T}$ real multiplications must be added to compute the channelized energy detection and the channelized Teager-Kaiser detection respectively.

Fig. 6 shows the complexity of each option as a function of the FFT size. For the channelized structure, two rejection requirements are used: $A=10 \mathrm{~dB}$ and $A=30 \mathrm{~dB}$. It is obvious that the time domain algorithms have lower complexity when no channelization is done. However, the results underline that the proposed FFT based detector has a lower complexity compared with the filterbank based architecture.

Furthermore, this study doesn't take into account the fact that the FFT operation could be performed with the same FFT operation as the demodulation scheme. In that case, the FFT comes for free and the frequency domain detectors have nearly the same complexity as their time domain counterpart.

\section{CONCLUSIONS}

In this paper, the detection of narrowband wireless microphone signals has been addressed. The motivation of this study was to identify a wireless microphone detector that could be implemented with an acceptable complexity. This paper 
focused on the complexity-performance tradeoffs of the algorithms and two main conlusions can be drawn. First, wideband signal may be monitored using FFT operation. Since the FFT implementation may be shared between communication and sensing schemes, the approach leads to a low complexity detector. In the wireless microphone study case, the proposed architecture brings a gain of $6 \mathrm{~dB}$ gain compared to time domain detectors. Second, the computation of the TeagerKaiser energy operator may favorably be implemented in the frequency domain and brings a gain of $1.5 \mathrm{~dB}$ compared to frequency domain energy detection.

Future work will be dedicated to the study of a detection threshold calibration. Then, a hardware implementation of the proposed algorithms and experimental measurements should be realized.

\section{ACKNOWLEDGMENT}

The research leading to these results was derived from the European Community's Seventh Framework Program (FP7) under Grant Agreement number 248454 (QoSMOS).

\section{REFERENCES}

[1] J. Mitola III and G. Q. Maguire Jr, "Cognitive radio: making software radios more personal," IEEE Personal Communications, vol. 6, no. 4, pp. 13-18, 1999.

[2] Official announcement of FCC, "FCC adopts rules for unlicensed use of television white spaces," available from http://www. ffC.gov, November 2008.

[3] H.-S. Chen, W. Gao, and D. Daut, "Spectrum sensing for wireless microphone signals," IEEE Sensor, Mesh and Ad Hoc Communications and Networks Workshops (SECONO8), June 2008.

[4] S. Xu, Y. Shang, and H. Wang, "SVD based Sensing of a Wireless Microphone Signal in Cognitive Radio Networks," IEEE International Conference on Communications Systems, November 2008.

[5] N. Han, S. H. Shon, J. O. Joo, and J. M. Kim, "Spectral correlation based signal detection method for spectrum sensing in IEEE 802.22 WRAN systems," International Conference on Advanced Communication Technology, February 2006.

[6] M. Ghosh, V. Gaddam, G. Turkenich, and K. Challapali, "Spectrum Sensing Prototype for Sensing ATSC and Wireless Microphone Signals," International Conference on Cognitive Radio Oriented Wireless Networks and Communications (CROWNCOMO8), May 2008.

[7] T. A. Weiss and F. K. Jondral, "Spectrum pooling: an innovative strategy for the enhancement of spectrum efficiency," IEEE Communications Magazine, March 2004.

[8] D. Noguet, M. Gautier, and V. Berg, "Advances in Opportunistic Radio Technologies for TVWS," EURASIP Journal on Wireless Communications and Networking, November 2011.

[9] M. Gautier, M. Laugeois, and D. Noguet, "Teager-Kaiser energy detector for narrowband wireless microphone spectrum sensing," IEEE International Conference on Cognitive Radio Oriented Wireless Networks and Communications (CROWNCOM), June 2010.

[10] A. Bouzegzi, P. Jallon, and P. Ciblat, "A second order statistics based algorithm for blind recognition of OFDM based systems," IEEE Global Communications Conference (GLOBECOM2008), December 2008.

[11] H. Urkowitz, "Energy detection of unknown deterministic signals," Proceeding of the IEEE, vol. 55, no. 4, pp. 523-531, April 1967.

[12] A. Sonnenschein and P. M. Fishman, "Radiometric detection of spreadspectrum signals in noise of uncertain power," IEEE Transactions on Aerospace and Electronic Systems, vol. 28, no. 3, July 1992.

[13] S. J. Shellhammer, "Spectrum sensing in IEEE 802.22," IAPR Workshop on Cognitive Information Processing, June 2008.

[14] J. F. Kaiser, "On simple algorithm to calculate the energy of signal," IEEE International Conference on Acoustics, Speech, and Signal Processing (ICASSP90), April 1990.

[15] A. Akansu and R. Haddad, Multiresolution Signal Decomposition: Transforms, Subbands and Wavelets, Academic Press, 2001. 